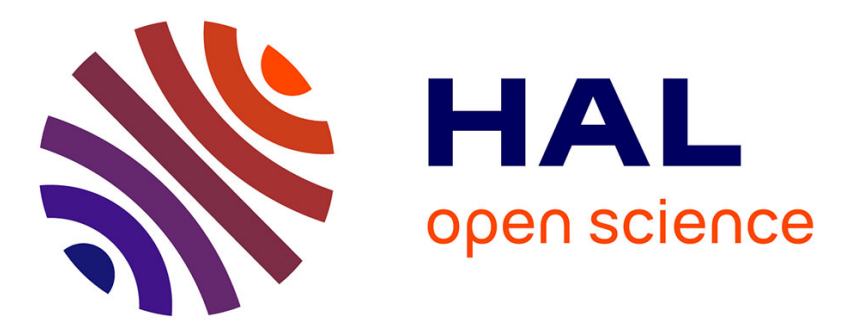

\title{
Controlled allylation of polyelectrolytes: a deep insight into chemical aspects and their applicability as building blocks for robust multilayer coatings
}

Thi-Thanh-Tam Nguyen, Sabrina Belbekhouche, Rémi Auvergne, Benjamin Carbonnier, Daniel Grande

\section{To cite this version:}

Thi-Thanh-Tam Nguyen, Sabrina Belbekhouche, Rémi Auvergne, Benjamin Carbonnier, Daniel Grande. Controlled allylation of polyelectrolytes: a deep insight into chemical aspects and their applicability as building blocks for robust multilayer coatings. Pure and Applied Chemistry, 2019, 91 (6), pp.983-995. 10.1515/pac-2018-1104 . hal-02157150

\author{
HAL Id: hal-02157150 \\ https://hal.science/hal-02157150
}

Submitted on 5 Jul 2021

HAL is a multi-disciplinary open access archive for the deposit and dissemination of scientific research documents, whether they are published or not. The documents may come from teaching and research institutions in France or abroad, or from public or private research centers.
L'archive ouverte pluridisciplinaire HAL, est destinée au dépôt et à la diffusion de documents scientifiques de niveau recherche, publiés ou non, émanant des établissements d'enseignement et de recherche français ou étrangers, des laboratoires publics ou privés. 


\title{
Controlled allylation of polyelectrolytes: a deep insight into chemical aspects and their applicability as building blocks for robust multilayer coatings
}

\author{
Thi-Thanh-Tam Nguyenª, Sabrina Belbekhouchea, Rémi Auvergne ${ }^{\mathrm{b}}$, Benjamin \\ Carbonniera, and Daniel Grande ${ }^{\star a}$
}

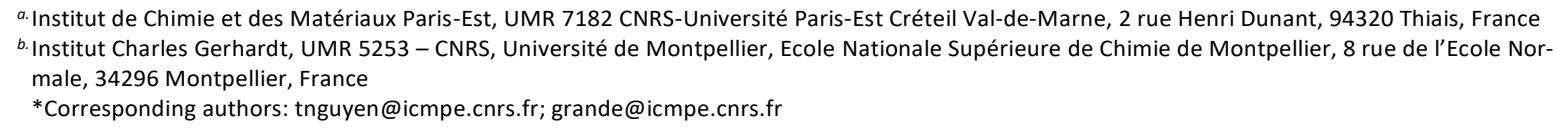

a. Institut de Chimie et des Matériaux Paris-Est, UMR 7182 CNRS-Université Paris-Est Créteil Val-de-Marne, 2 rue Henri Dunant, 94320 Thiais, France b. Institut Charles Gerhardt, UMR 5253 - CNRS, Université de Montpellier, Ecole Nationale Supérieure de Chimie de Montpellier, 8 rue de l'Ecole Normale, 34296 Montpellier, France

*Corresponding authors: tnguyen@icmpe.cnrs.fr; grande@icmpe.cnrs.fr

\begin{abstract}
:
Polyelectrolytes (PEs) bearing easily derivatizable functions for possible post-modification under mild conditions can find a broad range of applications in various fields. The present paper describes the successful controlled side-chain allylation of two types of PEs: polyamine-based polycations, i.e. poly(allylamine hydrochloride) (PAH) and branched polyethyleneimine (PEI), and strong polyanions, i.e. poly(sodium vinyl sulfonate) (PVS) and poly(sodium 4-styrene sulfonate) (PSS). PSS has been largely investigated in the literature, while PVS is much less commonly explored. The allylation of each type presents its own drawback, i.e. heterogeneous reaction in the case of strong polyanions and instability of partially protonated allylated polyamine products. Nevertheless, all encountered difficulties could be solved and thoroughly elucidated by different experimental tests.

This partial allyl-functionalization does not affect the electrolytic properties of the newly allylated PEs, as evidenced by the effective construction of two series of polyelectrolyte multilayer (PEM) films, namely PEI-ene(PSSene/PAH-ene $)_{4}$ and PEI-ene(PVS-ene/PAH-ene) $)_{4}$, the latter being one of the rare examples developed in the literature. The presence of allyl groups on the PE side-chains allows for the stabilization of the resulting PEM films via thiol-ene photo-crosslinking in the presence of a water-soluble dithiol crosslinker. In order to fix permanently the resulting crosslinked PEM films on substrates, the covalent crosslinking occurs not only between different $\mathrm{C}=\mathrm{C}$ bonds on $\mathrm{PE}$ layers but also with those present on substrates preliminarily functionalized with allyl groups via sulfur-gold chemistry. The robustness of both resulting crosslinked PEM films under strongly basic solution ( $\mathrm{pH} 14$ ) is validated by Quartz Crystal Microbalance (QCM) measurements. The versatility and effectiveness of the present approach is expected to find potential applications in different scientific and technological fields.
\end{abstract}

Keywords: allylation, polyelectrolyte, strong polyanion, hydroamination, covalent crosslinking, Layer-by-Layer (LbL) assembly 


\section{Introduction}

Polyelectrolytes (PEs), known as charged polymers, are still of major interest in polymer chemistry and materials science, mainly owing to their water solubility properties that allow them to find applications in different areas, including cosmetics, [1] concrete and cement formulations[2, 3], water treatment [4-6], drug delivery,[7-9], tissue engineering [10-12], optoelectronics,[13] and surface coatings, especially via alternative adsorption of polycations and polyanions to form polyelectrolyte multilayer (PEM) films.[14-18]

The so-called Layer-by-Layer (LbL) technique allows films to be built-up with tunable properties in terms of chemical nature, thickness, swelling behavior etc. Nevertheless, the stability of the resulting films relies on electrostatic interactions between the adsorbed PE layers, making the multilayer coatings sensitive to variations of $\mathrm{pH} /$ ionic strength or presence of polar solvent.[19] To overcome such an instability that is detrimental to industrial and biomedical end-uses subjected to environmental changes, different covalent crosslinking approaches have been reported [2022], namely via: (i) amide bond formation between complementary groups located on adjacent native PE layers via either carbodiimide chemistry, $[23,24]$ or heating $[25,26]$, (ii) "click" reactions between different PE layers bearing clickable side-groups [27-29], (iii) disulfide bond formation between different PE layers bearing thiol groups [30], (iv) imine bond formation between polyamines and bifunctional molecules [9, 31, 32] and finally, (v) photo-induced crosslinking between a diazido crosslinker and different layers of native PEs.[33]

Considerable effort has been focused on introducing reactive groups onto PE main chains for various advanced applications.[34-38] Indeed, the presence of such functions would enable PEs to act as reactive substances capable of undergoing a definite chemical action on one or more desired components in a surrounding medium but not merely as inert materials. We have recently reported a controlled side-chain allylation of two polycations, namely poly(allylamine hydrochloride) (PAH), branched polyethyleneimine (PEI), and especially that of a strong polyanion, i.e. poly(sodium 4-styrene sulfonate) (PSS).[39] Unlike weak polyanions [27, 28], allyl-functionalization of PSS had not been reported before, while this polyanion displays permanent negative charges of sulfonate groups even under acidic conditions, leading to a broader range of applications. [40, 41] The dual functionality of the resulting allylated PEs-ene, i.e. polyelectrolyte nature in tandem with side allyl groups, enabled their multilayer self-assembly through LbL deposition as well as the subsequent stabilization of the films through crosslinking with a water-soluble macromolecular dithiol crosslinker (Fig. 1). It is noteworthy that this original approach enabled to prepare robust PEM films without any limitation found in the aforementioned approaches reported in the literature, i.e. (i) heating, not always possible depending upon the nature of the substrate, (ii) highly air-sensitive thiolated PEs (thiols easily oxidized into disulfide $\mathrm{S}-\mathrm{S}$ or even into sulfonate $\mathrm{SO}_{3}{ }^{-}$), (iii) limited crosslinker (glutaraldehyde crosslink only between polyamines (polycations) while polyanions are still free), and most importantly (iv) delamination of crosslinked PEM films from the substrate, owing to the absence of covalent bonds between the latter and the crosslinked PEM films.

Besides the success of forming robust PEM films using partially allylated PEs, some chemical issues were encountered during the allylation of each PE type, especially the chemical instability of the partially protonated allylated polyamine-based polycations under some specific conditions. In this context, the present investigation first aims at thoroughly elucidating the reaction mechanism inducing such an unexpected instability, and second at extending our approach to a less commonly investigated strong polyanion, i.e. poly(sodium vinyl sulfonate) (PVS). PVS has been selected not only because its LbL self-assembly has not been fully explored so far [42, 43] but also because it can be used as a heparin-mimicking polymer [44], thus providing potential biological applications to the newly developed coatings. 


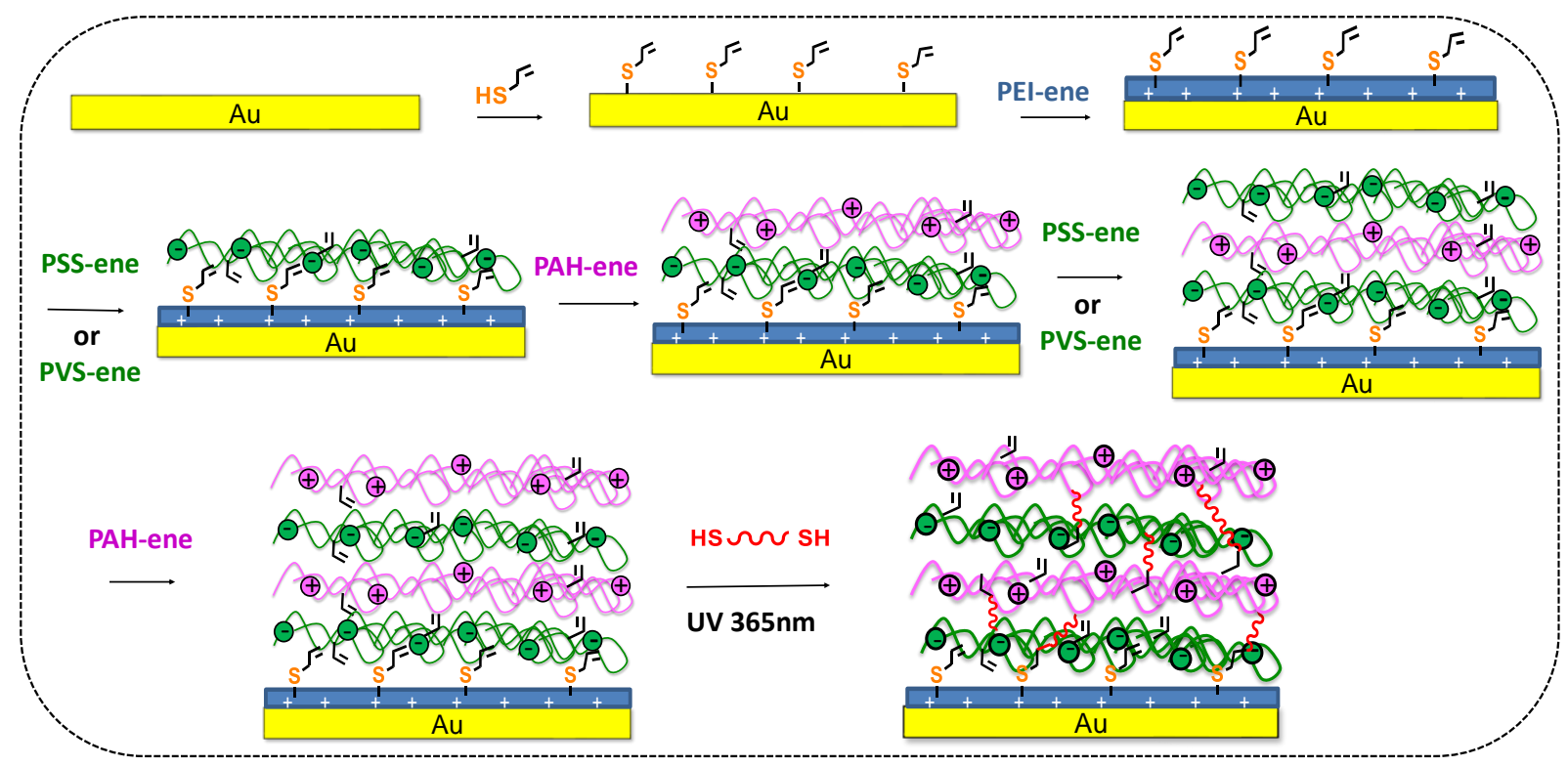

Fig. 1: Scheme describing the approach toward enhancing the stability of PEM films on gold substrates.

\section{Materials and methods}

\subsection{Materials}

Poly(allylamine hydrochloride) (PAH, $M_{\mathrm{w}} \sim 50000 \mathrm{~g} \cdot \mathrm{mol}^{-1}$ ), allyl bromide (99 \%), and triethylamine (TEA, $99 \%$ ), were purchased from Alfa Aesar. Poly(allylamine) $\left(M_{\mathrm{w}} \sim 65000\right.$ g. $\mathrm{mol}^{-1}, 10$ wt. $\%$ in water), poly(4-styrene sulfonic acid) $\left(M_{\mathrm{w}} \sim 75000\right.$ g. $\mathrm{mol}^{-1}, 18$ wt. $\%$ in water); branched polyethyleneimine (PEI, $M_{\mathrm{n}} \sim 10000$ g.mol ${ }^{-1}, M_{\mathrm{w}} / M_{\mathrm{n}}=2.5$ ), poly(sodium vinyl sulfonate) (PVS, $M_{\mathrm{w}} \sim 5000 \mathrm{~g} \cdot \mathrm{mol}^{-1}$ ), poly(ethylene glycol) (PEG, $M_{\mathrm{w}} \sim 1000 \mathrm{~g} \cdot \mathrm{mol}^{-1}$ ), sodium hydroxide, and 2-propene-1-thiol were purchased from Sigma Aldrich. Thionyl chloride (99\%) was purchased from Fluka. Dimethylformamide (DMF), dichloromethane (DCM), tetrahydrofuran (THF), ethanol, and acetone were purchased from SDS Carlo Erba. Reagents and solvents were used without any further purification, unless otherwise stated. Water was purified with a Milli-Q reagent system (Millipore).

\subsection{Modification of gold substrates}

\subsubsection{Pre-treatment of flat gold substrate}

The gold-coated QCM crystal was first cleaned with water and ethanol, then treated in a UV/ozone chamber (BioForceUV/OzoneProCleaner) for $20 \mathrm{~min}$. After an additional rinsing with ethanol, surfaces were dried under $\mathrm{N}_{2}$ flow. The cleaned substrate was introduced into a solution of 2-propene-1-thiol ( $1 \mathrm{~g} . \mathrm{L}^{-1}$ in ethanol) for $24 \mathrm{~h}$ at $25^{\circ} \mathrm{C}$. The substrate was then taken out and rinsed abundantly with ethanol. The substrate was again immersed into a PEI-ene solution ( $1 \mathrm{~g} . \mathrm{L}^{-1}$ in water) adjusted to $\mathrm{pH} 3$ for $24 \mathrm{~h}$ at $25^{\circ} \mathrm{C}$. These steps enabled to anchor a primer layer (PEI-ene) for better adsorption of PEM films, i.e. PEI-ene(PSS-ene/PAH-ene) $)_{n}$ or PEI-ene (PVS-ene/PAH-ene) $)_{n}$ where $n$ stands for the number of bilayers.[14]

\subsubsection{Elaboration of PEI-ene(PSS-ene/PAH-ene) ${ }_{n}$ or PEI-ene(PVS-ene/PAH-ene)n multilayered films}

After mounting in the QCM flow chamber, the sensor was conditioned in acidic medium ( $\mathrm{pH} 3$ ) for 10 min. The self-assembly of PSS-ene/PAH-ene or PVS-ene/PAH-ene was performed onto the pre-treated flat gold substrate through the alternative contact with the PSS-ene or PVS-ene and PAH-ene solutions (1 g.L ${ }^{-1}$ adjusted to $\left.\mathrm{pH} 3\right)$ for 15 min, followed by a thorough rinsing with Milli-Q water adjusted to $\mathrm{pH} 3$ for approximately $10 \mathrm{~min}$ after each contact with PE-ene solutions. Polymer solutions were injected into the cell with a flow rate of $500 \mu \mathrm{L} \cdot \mathrm{min}^{-1}$ at $25^{\circ} \mathrm{C}$. 


\subsubsection{Photochemical crosslinking of polyelectrolyte multilayer films}

The gold substrate with PEI-ene(PSS-ene/PAH-ene) ${ }_{n}$ or PEI-ene(PVS-ene/PAH-ene) $)_{n}$ multilayers on top was immersed into a $25 \mathrm{mM}$ aqueous solution of PEG-based dithiol (PEG-diSH) and Irgacure 2959 (1 mol \%). The system was irradiated during $30 \mathrm{~min}$ at $365 \mathrm{~nm}$ in a UV oven $(6 \mathrm{x} 15 \mathrm{~W})$. The PEG-diSH synthesis was reported in our previous work.[39]

\subsection{Instrumentation}

${ }^{1} \mathrm{HNMR}$ and ${ }^{13} \mathrm{CNMR}$ spectra were recorded on a Bruker Avance III spectrometer with an operating frequency of $400 \mathrm{MHz}$ and $100 \mathrm{MHz}$, respectively.

FT-IR spectra were recorded on a Nicolet Magna-IR 550 spectrometer using the attenuated total reflection (ATR) mode between 4000 and $450 \mathrm{~cm}^{-1}$ with an average of 32 consecutive scans and a resolution of $4 \mathrm{~cm}^{-1}$.

$\zeta$-Potential values were determined on a Zetasizer Nano-ZS (Zetasizer 4700 Malvern Instruments, Brookheaven Instruments Corporation, USA) equipped with a He-Ne laser $\left(\lambda=633 \mathrm{~nm}\right.$; scattering angle: $\left.173^{\circ}\right)$

A Quartz Crystal Microbalance (QCM) was employed for monitoring the formation of PEM films and their stability. A Q-Sense E1 (Q-Sense, Sweden) system equipped with gold-coated quartz crystal sensors (Lot-Oriel, France) with a nominal frequency of about $5 \mathrm{MHz}$ was used.

\section{Results and discussion}

\subsection{Functionalization of polyelectrolytes}

The goal of this study was to introduce functional groups into polyelectrolyte side-chains while maintaining their electrolytic properties, i.e. water solubility and complexation ability with PEs of opposite charge to sustain the LbL process. These properties are essential for their subsequent utilization in the construction of PEM films. Allylic moieties were chosen as functional side groups, since they were reactive for further chemical crosslinking under mild condition of thiol-ene "click" reaction in the presence of a suitable dithiol crosslinker. Moreover, the air stability of these functional groups would allow for an easy handling of the resulting allylated PEs without any risk of oxidation, problem usually met in thiolated PEs [27, 36, 45]. Two couples of polyelectrolytes PSS/PAH and PVS/PAH were chosen for this study: the first one has been known for producing PEM films with high performance,[46] while the second one has sparsely been investigated [42]. Consequently, two conventional polycations (branched PEI and PAH) and two strong polyanions (PSS and PVS) were subjected to controlled side-chain allylation. The allyl-functionalization of branched PEI (PEI-ene) was necessary for initial adsorption of the first layer on surface. Indeed, this primer layer was

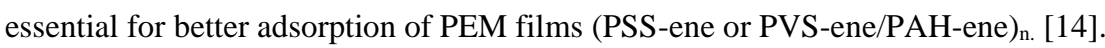

\subsection{Allyl-functionalization of polycations and investigation of chemical instability of resulting allylated polyamines}

Amines can act either as a nucleophile or as a base. An aliphatic polyamine could be assumed to undergo reactions similar to that of a monoamine but with steric hindrance effects potentially caused by polymer chains. In this regard, the partially allylated PAH-ene or PEI-ene were accessible via nucleophilic substitution between allyl bromide (electrophile) and neutral poly(allylamine) (PAH) (Fig. 2, bottom, route 1) or neutral branched PEI (Fig. 2, top) as "polynucleophiles", respectively. The presence of multiple amino functions on polymer chains enabled these polyamines to play also the role of a "polybase" to entrap $\mathrm{HBr}$ generated from nucleophilic substitution reaction, thus providing partially protonated allylated polyamines intermediates $\mathbf{1}$ and $\mathbf{2}$. The allylation ratio of polyamines could be controlled simply by adjusting the added amount of allyl bromide, since both neutral polyamines and allyl bromide were well soluble in the reaction mixture. 


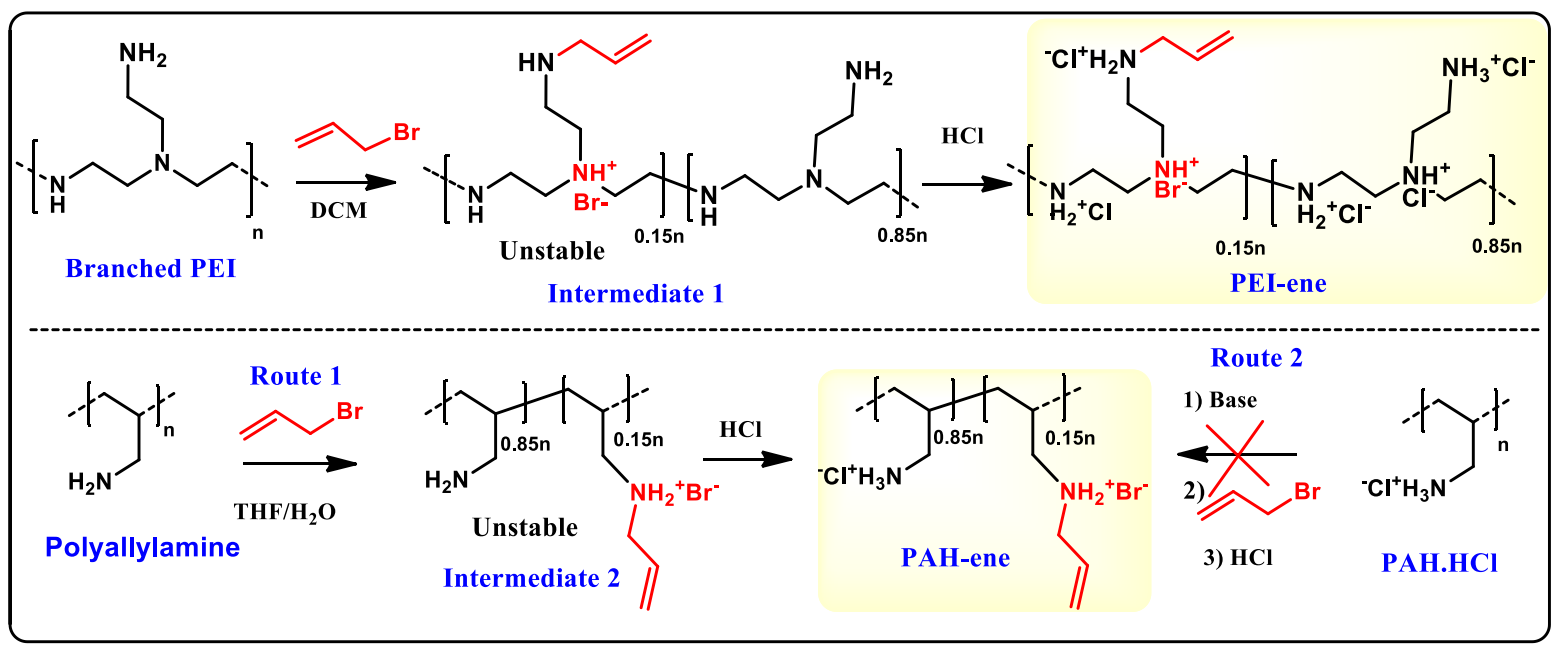

Fig. 2: Partial allylation of $\mathrm{PEI}$ and $\mathrm{PAH}$.

It is worth mentioning that we had initially started the allylation of poly(allylamine) in its ammonium salt form (PAH.HCl) (Fig.2, bottom, route 2). Due to the non-nucleophilicity of the ammonium side groups, before introducing the electrophilic species into the reaction mixture, a reasonable amount of triethylamine (TEA) was added to generate highly nucleophile $-\mathrm{NH}_{2}$ function that reacted easily with allyl bromide to give the desired PAH-ene in the presence of triethylammonium chloride salt as a side-product. Unfortunately, the latter could not be efficiently separated from PAH-ene either by precipitation or by dialysis against water. Indeed, due to the similar solubility behavior of PAH-ene and this side-product, the purification by precipitation was impossible. In the case of dialysis, despite the smallest molecular weight cutoffs of the dialysis tubing used $(\mathrm{MWCO}=500 \mathrm{Da})$, almost $97 \%$ of PAH-ene $\left(M_{\mathrm{w}} \sim 56000 \mathrm{~g} \cdot \mathrm{mol}^{-1}\right)$ passed through their membrane together with triethylammonium chloride salts at neutral $\mathrm{pH}(\mathrm{pH}$ at which the dialysis tubing is chemically stable). This phenomenon could be explained as follows: at pH 7, most of amino side-groups were under their protonated forms and thus PAH-ene bearing multiple positive charges on its side-chain would adopt an unfolded conformation. This conformation would allow it to pass easily through the dialysis tubing even with the smallest MWCO. This issue of purification prompted us to employ the poly(allylamine) in its neutral form as starting material (Fig. 2, bottom, route 1). After reacting with a desirable amount of allylbromide, the partially protonated intermediate 2 was acidified with an appropriate amount of $\mathrm{HCl}$ solution. This two-step procedure enabled to prepare stable allylated polyamines under their completely protonated forms (PEI-ene and PAH-ene).

It is important to stress that intermediates $\mathbf{1}$ or $\mathbf{2}$ exhibited a modulated solubility behavior in organic solvents. Indeed, $\mathrm{HBr}$ generated from the nucleophilic substitution between allyl bromide and amino groups on neutral polyamines instantly protonated these intermediates. Such a protonation increased significantly their polarity, leading to a pronounced decrease in their organo-solubility as compared to the starting neutral polyamines. Logically, such a decrease in organo-solubility was proportional to the amount of $\mathrm{HBr}$ generated, namely the degree of allylation (named DA): the higher the DA, the lower the organo-solubility of the partially allylated polyamine-based products. Indeed, it was found that the intermediate 1 or $\mathbf{2}$ with $\mathrm{DA} \geq 0.5$ presented a very low solubility in the reaction mixture (dichloromethane). Accordingly, in this study, both polyamines were chosen to be allylated at a moderated DA, i.e. $15 \%$. Surprisingly, at this DA, both intermediates 1 or $\mathbf{2}$ (under their anhydrous forms) exhibited an unstable behaviour of solubility upon standing at room temperature. Such an instability was as a function of time. Initially, they were completely soluble in water and in most of polar organic solvents, but after a few weeks standing at room temperature, their solubility reduced significantly with the formation of hydrogels observed in the presence of water, and then beyond one month, they became completely insoluble in any solvent. Such a gradual change in solubility issues could be attributed to either (i) hydrogen bonding between protons on protonated amino groups and free doublet on neutral amino groups (hypothesis 1, Fig. 3A) or (ii) gradual hydroamination crosslinking between the activated double bond $\mathrm{C}=\mathrm{C}$ (nearby a protonated amino group) and a neutral amino group (hypothesis 2, Fig. 3B) on the polymer side-chains. It should be stressed that these two possible phenomena could happen only when partially protonated allylated polyamines were involved (case of intermediates $\mathbf{1}$ and $\mathbf{2}$ ). 


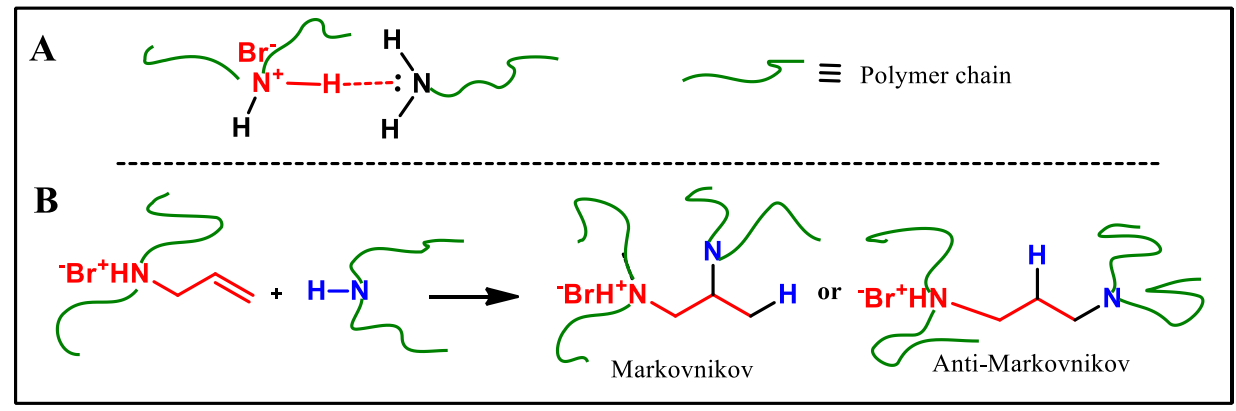

Fig. 3: Hypotheses suggesting two possible reasons provoking the instability of intermediates 1 and 2: (A) hydrogen bonding between hydrogens of protonated amino groups and free doublet on neutral amino groups; (B) hydroamination between an activated double bond and a neutral amine.

To verify the first hypothesis, a simple experimental test was performed as follows: an aqueous solution of neutral PEI was partially protonated (15\% compared to the number of repetitive monomer units) using $\mathrm{HCl}$ aqueous solution. After completely removing water, the resulting partially protonated PEI was stored in a closed vial at room temperature (same conditions inducing the insolubility of intermediates $\mathbf{1}$ and $\mathbf{2}$ ), and then its water solubility was assessed as a function of time. Under these conditions, we observed a gradual decrease in the latter characteristic, mostly beyond one month. However, unlike the case of partially allylated PEI (intermediate 1), the non-allylated one still remained partially soluble after a long period of stirring (a few days to a few weeks). Therefore, the complete insolubility of the intermediate 1 after standing at least one month at room temperature could be attributed only partially (but not completely) to the occurrence of hydrogen bonding between hydrogen on ammonium groups and free doublet on neutral amino ones.

As the full reason for the complete insolubility of intermediates $\mathbf{1}$ or $\mathbf{2}$ was not fully identified, the second hypothesis was also verified. To this purpose, the water solubility at room temperature of nine samples of dry intermediate $\mathbf{1}$ $(\mathrm{DA}=15 \%)$ adjusted at nine different $\mathrm{pH}$ values, i.e. $1,5,7,8,8.5,9,10,11$, and 12 , was assessed as a function of time. Among these samples, only those whose $\mathrm{pH}$ was adjusted between 8 and 11 exhibited a slight change in color (from white to yellowish), and most importantly a gradual decrease in their water solubility mostly beyond one month standing at room temperature, while no change was observed for samples adjusted at other $\mathrm{pH}$ ranges. It is well known that $\mathrm{pKa}$ values of amine species (primary, secondary and tertiary) present in the intermediate $\mathbf{1}$ range from 9 (named $\mathrm{pKa}_{\min }$ ) to around 10 (named $\mathrm{pKa}_{\max }$ ). It means that both protonated and neutral amine species would coexist in the $\mathrm{pH}$ range from $\mathrm{pKa}_{\min }-1$ (i.e., 8) to $\mathrm{pKa}_{\max }+1$ (i.e., 11). Such a coexistence of two amino species would induce the hydroamination crosslinking as suggested in the second hypothesis (Fig. 3B). Indeed, when a protonated amino group was connected to an allylic moiety, the double bond $\mathrm{C}=\mathrm{C}$ of the latter would be activated due to an electronwithdrawing inductive effect (-I effect) of the positively charged nitrogen atom. The remaining neutral amino groups would react with such activated $\mathrm{C}=\mathrm{C}$ bonds according to an addition mechanism. That is the reason why no insolubility problem was observed in the case of completely protonated $(\mathrm{pH} \leq 8)$ or completely neutral $(\mathrm{pH} \geq 11)$ allylated polyamines.

Chemical characterization by solid-state NMR $\left({ }^{1} \mathrm{H} \&{ }^{13} \mathrm{C}\right)$ and by FTIR-ATR was carried out but failed to provide an evidence for the hydroamination crosslinking occurring on intermediates $\mathbf{1}$ and $\mathbf{2}$. This was because not all double bonds were consumed after crosslinking, and most importantly no new function was generated (only amino function present before and after crosslinking). To overcome this difficulty of chemical characterization, a model reaction between small molecules, namely $n$-butylamine and allyl bromide, was performed (Fig. 4). This model reaction would enable to use liquid ${ }^{1} \mathrm{H}$ NMR to quantify an eventual decrease in intensity of allylic protons after hydroamination reaction. To make sure that identical conditions inducing the insolubility of allylated polyamines were applied, this model reaction was performed upon using only 0.2 equivalent of allyl bromide at room temperature in the absence of solvent. 


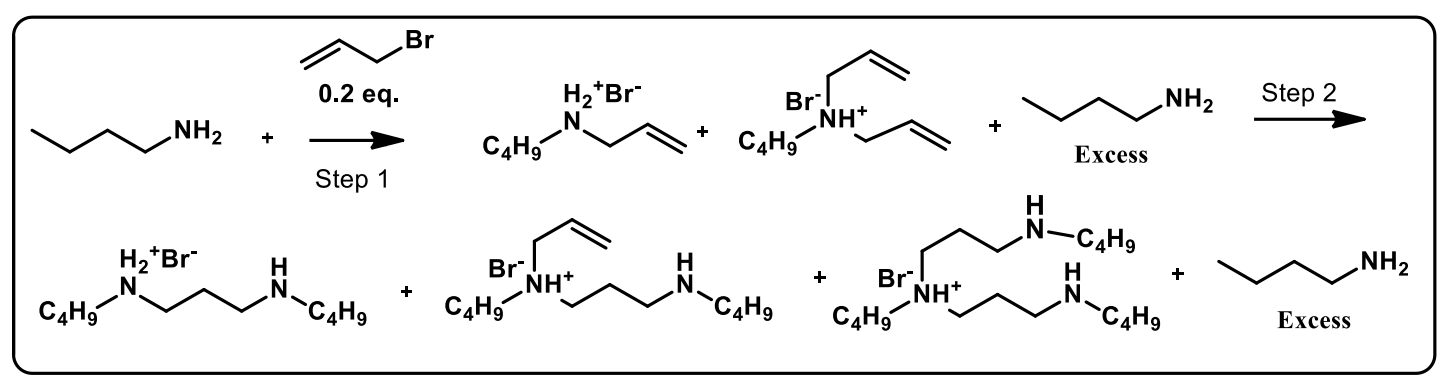

Fig. 4: Model reaction between $n$-butyl amine and allyl bromide (step 1); Hypothesis of reactions between different products from step 1 (step 2).

As shown in Fig. 4, each nucleophilic substitution reaction between an amine and an alkylating agent (allyl bromide) generates one $\mathrm{HBr}$ molecule. Such reactions are difficult to control because the resulting secondary amines are often more nucleophilic than their precursors, and thus will further react with the alkylating agent yielding ternary amines. In principle, the generated $\mathrm{HBr}$ would preferentially protonate secondary or tertiary amines instead of the initial primary ones because the basicity of the latter is lower. As mentioned above, after nucleophilic substitution (step 1), the double bond of allylic moieties linked to the positively charged ammoniums should be activated and would undergo the hydroamination with the remaining neutral amino groups (step 2). Because of the large size of $-\mathrm{NHC}_{4} \mathrm{H}_{9}$ group (compared to that of proton) and the electron-withdrawing inductive effect (-I effect) of the positively charged nitrogen atom nearby $\mathrm{C}=\mathrm{C}$ double bond, a regioselective hydroamination would occur and thus anti-Markovnikov products were supposed to be formed essentially. These two steps would generate a mixture of many different products whose ${ }^{1} \mathrm{H}$ NMR spectra would be difficult to be interpreted. Indeed, the diversity of products was confirmed by the presence of up to four different ${ }^{1} \mathrm{H}$ NMR signals of $-\mathrm{CH}_{2}$ - nearby amino groups, i.e. 3.30, 3.05, 2.76, and $2.63 \mathrm{ppm}$ (Fig. 5). The first two ones could be ascribed to those nearby positively charged amino (secondary and ternary ones), while the last two ones could be assigned to those nearby neutral amino groups (secondary and primary ones). Despite of this structural complexity, the gradual hydroamination between products from step 1 could be easily evidenced by a gradual decrease in the intensity of allylic protons (between 5 and $6 \mathrm{ppm}$ ) as a function of time. At the beginning, i.e. immediately after mixing $n$-butylamine and allyl bromide $\left(\mathrm{t}_{0}\right)$, only signals arising from both reactants were detected. However, beyond $16 \mathrm{~h}$, the whole spectrum changed, i.e. (i) signals of free allyl bromide disappeared (6.05, 5.34, and $5.16 \mathrm{ppm}$ for three protons of $-\mathrm{CH}=\mathrm{CH}_{2}$ moiety and $3.95 \mathrm{ppm}$ for two protons of $-\mathrm{CH}_{2} \mathrm{Br}$ ), and new allylic protons signals appeared (5.90, 5.20, and $5.12 \mathrm{ppm}$ ), indicating a complete consumption of free allyl bromide (step 1), and (ii) a significant decrease in the intensity of allylic protons was observed, thus indicating that the hydroamination effectively occurred and the most pronounced effect was observed after 60 days (step 2). 


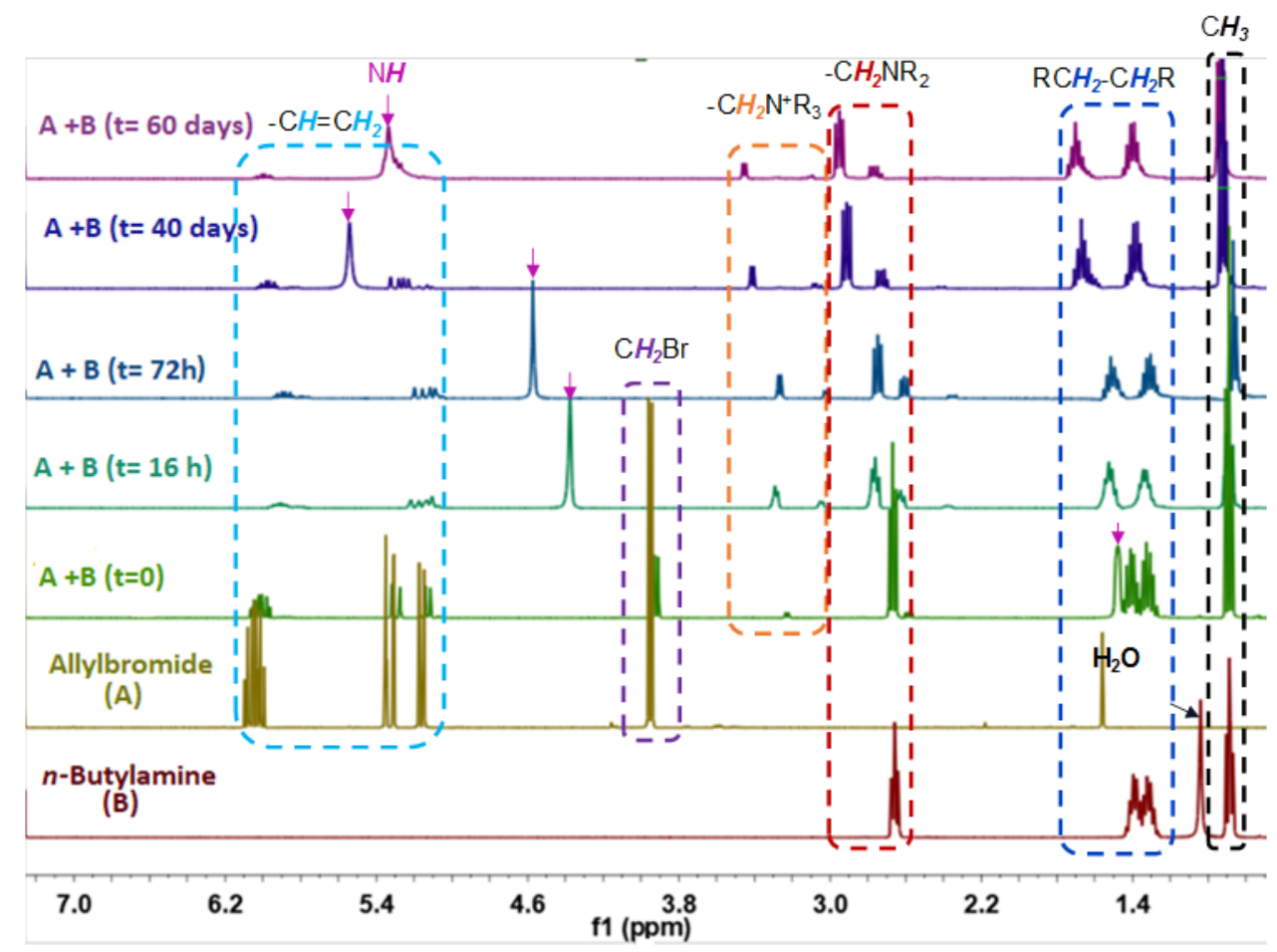

Fig. 5: ${ }^{1} \mathrm{H}$ NMR study on a model reaction between $n$-butylamine and allyl bromide for evidencing hydroamination. The arrows indicate the chemical shift of labile protons on protonated ammonium groups.

Accordingly, both above mentioned hypotheses were proved. This meant that the instability of the partially protonated allylated polyamines was arisen not only from hydrogen bonding (physical interactions) but also from hydroamination (chemical bonding). The latter reaction could be considered as the main reason that induced a permanent insolubility of intermediates 1 or $\mathbf{2}$ (Fig. 2), owing to the covalent nature of the formed bonds. Fortunately, such instability issues could be solved easily upon storing the resulting allylated polyamines under their fully protonated forms, namely PEI-ene and PAH-ene, before use.

\subsection{Allyl-functionalization of strong polyanions}

The partial allylation of strong polyanion PSS has been recently reported by our group (Fig. 6, top).[39] Such a functionalization had not been reported previously, probably due to the limited solubility of this strong polyanion (only in water) and the unusual sulfonyl chemistry. The allyl-functionalization ratio should be moderated for maintaining the water solubility of the resulting allylated PSS (PSS-ene) because each sulfonamide bond formation neutralizes one negative charge of a sulfonate group. This procedure of preparing partially allylated PSS was successfully extended to its less rigid homologue PVS (Fig. 6, bottom). The latter was chosen not only because of its strong electrolytic properties, but also because of its ability of stimulating cell proliferation arisen from its diverse biological functions similar to the highly sulfated polysaccharide (Heparin), as reported recently by Nguyen et al.[[44]. Consequently, the resulting newly crosslinked PVS/PAH based-PEM films could be expected to be used in various biomedical applications. 


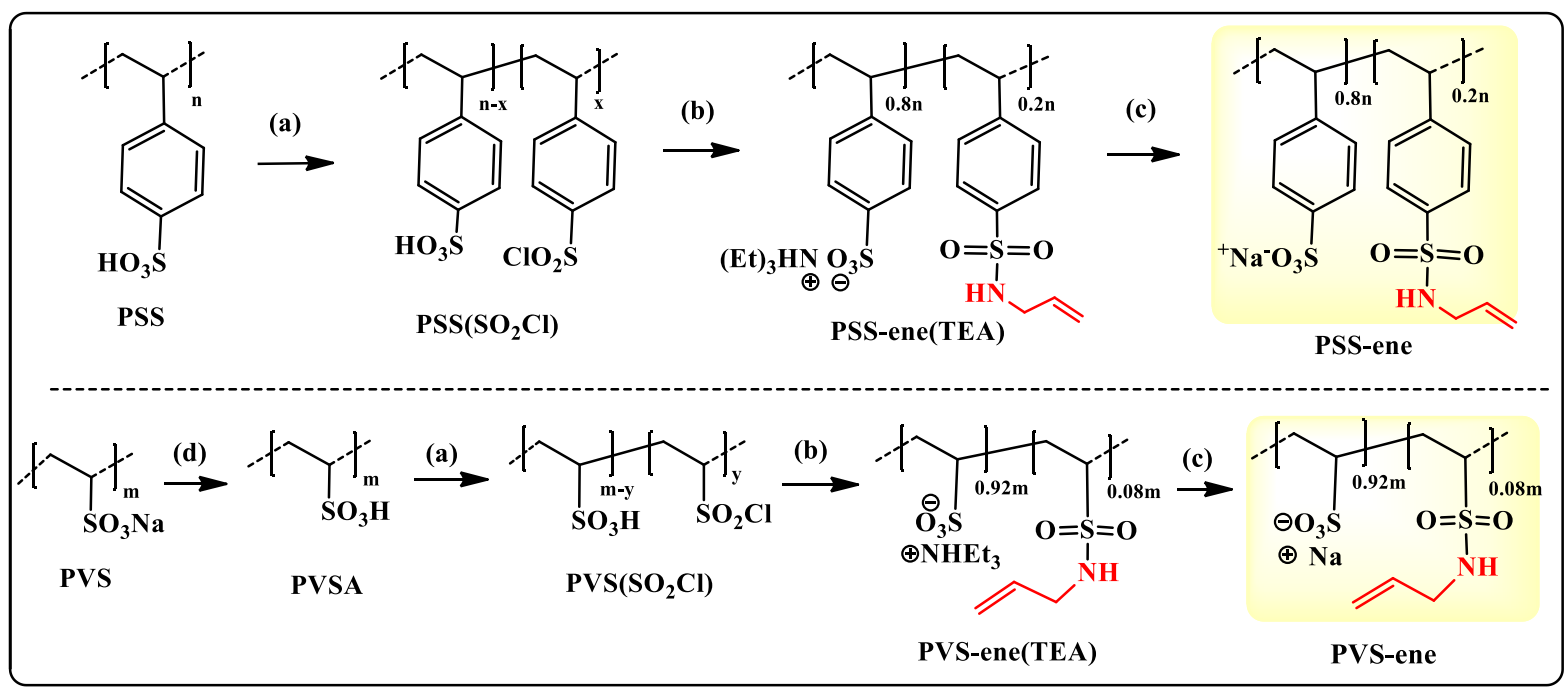

Fig. 6:Controlled allylation of PSS and PVS. Reaction conditions: (a) Excess of $\mathrm{SOCl}_{2}$, reflux (heterogeneous reaction); (b) step 1: TEA, DMF and step 2: allylamine (0.3 eq. vs. repeating monomer units); (c) step 1: $\mathrm{NaOH}_{\mathrm{aq}}$ and step 2: dialysis; $\left(\right.$ d) $\mathrm{HSO}_{3} \mathrm{Cl}$; Overall yields: $60 \%$ and $52 \%$ for PSS and PVS, respectively.

For possible chemical transformation, poly(vinyl sulfonic acid) (PVSA) had to be used as a starting material instead of PVS. The commercially available PVS solution was first acidified to generate PVSA together with $\mathrm{NaSO}_{3} \mathrm{Cl}$ (Fig. 6). The latter side-product was eliminated simply by dialysis against water. Before converting $-\mathrm{SO}_{3} \mathrm{H}$ side groups on PVSA into the activated intermediate sulfonyl chloride $\mathrm{PVS}\left(\mathrm{SO}_{2} \mathrm{Cl}\right)$ using thionyl chloride $\mathrm{SOCl}_{2}$ as a reagent, the water in pure PVSA solution had to be removed completely since its presence (even in trace amounts) would destroy the $\mathrm{SOCl}_{2}$ reagent. Furthermore, it was primordial to grind the solid PVSA into fine powder to increase the contact area between the insoluble powder of PVSA and $\mathrm{SOCl}_{2}$ reagent. Given an inhomogeneity of the reaction mixture, not all $\mathrm{SO}_{3} \mathrm{H}$ groups in PVSA were converted into $-\mathrm{SO}_{2} \mathrm{Cl}$ despite an excess of $\mathrm{SOCl}_{2}$ used. As a result, the intermediate $\mathrm{PVS}\left(\mathrm{SO}_{2} \mathrm{Cl}\right)$ bore two kinds of side-groups, namely $-\mathrm{SO}_{3} \mathrm{H}$ and $-\mathrm{SO}_{2} \mathrm{Cl}$. Before adding a desirable quantity of allylamine reagent, i.e. 0.3 equivalent, an excess of a base, i.e. triethylamine (TEA), had to be introduced for converting the remaining $-\mathrm{SO}_{3} \mathrm{H}$ functions into the protected form, namely triethylammonium sulfonate salts $-\mathrm{SO}_{3}{ }^{-} \mathrm{NHEt}_{3}{ }^{+}$. The latter could no longer react with free allylamine according to an acid-base reaction. In other words, the amount of allylamine added was restricted to react with $-\mathrm{SO}_{2} \mathrm{Cl}$ side groups to form sulfonamide bonds, thus providing PVS-ene(TEA) intermediates. Finally, the ammonium cation in the resulting PVS-ene(TEA) was exchanged by a smaller one $\left(\mathrm{Na}^{+}\right)$ simply by adding an appropriate amount of $\mathrm{NaOH}$ aqueous solution. This reaction yielded the desired PVS-ene, while releasing $\mathrm{NaCl}$ and TEA as a weaker base. These two side-products were separated from PVS-ene by dialysis against water. Under these conditions, the allylation ratio in PVS-ene was found to be $\sim 8 \%$, as confirmed by ${ }^{1} \mathrm{H}$ NMR.This ratio was lower than that expected ( $30 \%$ ) as 0.3 equivalent of allyl bromide was used. This discrepancy was certainlydue to the insolubility of intermediate $\mathrm{PVS}\left(\mathrm{SO}_{2} \mathrm{Cl}\right)$ which reduced the accessibility of allyl bromide to the reactive sites $\left(-\mathrm{SO}_{2} \mathrm{Cl}\right)$.

Careful structural and chemical characterizations of both allylated polyanions, PSS-ene[39] and PVS-ene, were performed using ${ }^{1} \mathrm{H}$ NMR, ${ }^{13} \mathrm{C}$ NMR and FT-IR. As compared to ${ }^{1} \mathrm{H}$ NMR spectrum of pure PVS, the occurrence of new signals from allylic protons between $5.3 \mathrm{ppm}$ and $6.0 \mathrm{ppm}$ in that of PVS-ene clearly evidenced the successful allyl-functionalization of PVS (see SI Fig. 1). Such broadened signals were easily distinguished from the very wellresolved ones present in free allylamine. Indeed, after covalently bonding to the polymer chains, the mobility of allylic fragment would reduce significantly. Such a decrease in mobility resulted in the signal broadening in ${ }^{1} \mathrm{HNMR} .{ }^{13} \mathrm{C}$ NMR spectroscopy was also used to confirm the chemical structure of PVS-ene (see SI Fig.1). As compared to the spectrum of unmodified PVS, three new bands at 46,116 and $134 \mathrm{ppm}$ were observed in that of PVS-ene. These new signals could be attributed to allylic carbon atoms in the structure of PVS-ene.

In addition to ${ }^{1} \mathrm{H}$ and ${ }^{13} \mathrm{C}$ NMR analyses, FT-IR was also used to investigate the changes in functional groups, i.e, $-\mathrm{SO}_{3}{ }^{-}$to $-\mathrm{SO}_{2} \mathrm{Cl}$, and then to $-\mathrm{SO}_{2} \mathrm{NH}$-allyl (see SI Fig. 2). Since only $8 \%$ of $\mathrm{SO}_{3}{ }^{-}$groups were converted into sulfonamide functions, characteristic bands of the remaining sulfonate function (namely asymmetric and symmetric stretch- 
ing of $\mathrm{S}=\mathrm{O}$ in sulfonate at 1440 and $1160 \mathrm{~cm}^{-1}$ respectively) were also present in the spectrum of $\mathrm{PVS}\left(\mathrm{SO}_{2} \mathrm{Cl}\right)$ and that of PVS-ene. It was noteworthy to mention that most of bands in the spectrum of $\mathrm{PVS}\left(\mathrm{SO}_{2} \mathrm{Cl}\right)$ became broader as compared to those of neat PVS. Especially, the presence of two new absorption bands at 870 and $570 \mathrm{~cm}^{-1} \mathrm{could}_{\text {be as- }}$ signed to C-S stretching and $\mathrm{SO}_{2}$ rocking in sulfonyl chloride compounds, respectively. In the case of PVS-ene, eight new characteristic bands at 3060, 1429, 1670 (shoulder), 950, 1516, 1368, 1324 and $880 \mathrm{~cm}^{-1}$ were observed. The first four bands could be attributed to $=\mathrm{C}-\mathrm{H}$ stretching, $=\mathrm{C}-\mathrm{H}$ bending, $\mathrm{C}=\mathrm{C}$ stretching and $\mathrm{C}=\mathrm{C}$ bending, respectively, thus evidencing the success of grafting allylic moieties onto PVS chains. The last four bands were characteristic of N-H bending, $\mathrm{S}=\mathrm{O}$ stretching, $\mathrm{S}-\mathrm{N}$ stretching and $\mathrm{S}-\mathrm{N}$ bending, respectively, thus confirming the formation of sulfonamide bonds between PVS and allylic moieties.

\subsection{Monitoring the formation and the robustness of PEM films on a flat gold surface by QCM measurements}

In the present strategy, the preliminary introduction of allyl groups onto the gold coated quartz substrate was required to ensure strong adhesion between the substrate and the PEM film after thiol-ene covalent crosslinking. This pre-treatment was achieved by employing the commercially available 2-propene-1-thiol via the well-known sulfurgold surface chemistry.[47] Afterward, the modified quartz substrate was dipped in a PEI-ene solution used as a primer anchoring layer enabling further adsorption of the polyanion at $\mathrm{pH} 3$.

Herein, the QCM technique was used to demonstrate the alternative adsorption of the polyelectrolytes onto the modified crystal sensors. This was reflected by changes in resonance frequency, $\Delta F$, due to the adsorbed polyelectrolyte multilayers (PEI-ene(PVS-ene/PAH-ene) $)_{n}$ or PEI-ene(PSS-ene/PAH-ene) $)_{n}$, including trapped water molecules on the modified gold QCM sensors.

As seen from Fig. 7a, a typical decrease in the frequency signal was observed upon the successive steps of PE-ene adsorption. It should be mentioned that upon rinsing, a slight increase in the frequency shift was observed, due to the removal of some weakly bound polyelectrolyte chains. These QCM profiles fully supported the successful electrostatically-driven construction of coatings containing 4 polyanion/polycation bilayers through a LbL process. The value of the final frequency shift depended on the nature of the polyanion. Indeed, the values were $-80 \pm 5 \mathrm{~Hz}$ and $-150 \pm 7 \mathrm{~Hz}$ for PEI-ene(PVS-ene/PAH-ene) ${ }_{4}$ and PEI-ene(PSS-ene/PAH-ene) $)_{4}$, respectively. Although conclusions could not be drawn from these simple adsorption monitoring experiments, the observed difference might be related to the differences in the average molar masses of polyanions (75000 g. $\mathrm{mol}^{-1}$ for PSS vs. $5000 \mathrm{~g} \cdot \mathrm{mol}^{-1}$ for PVS) and in their electrolytic properties ( $-60 \pm 2 \mathrm{mV}$ for PSS-ene $v s .-23 \pm 2 \mathrm{mV}$ for PVS-ene) as determined by zeta-potential measurements at pH 3.

(a)

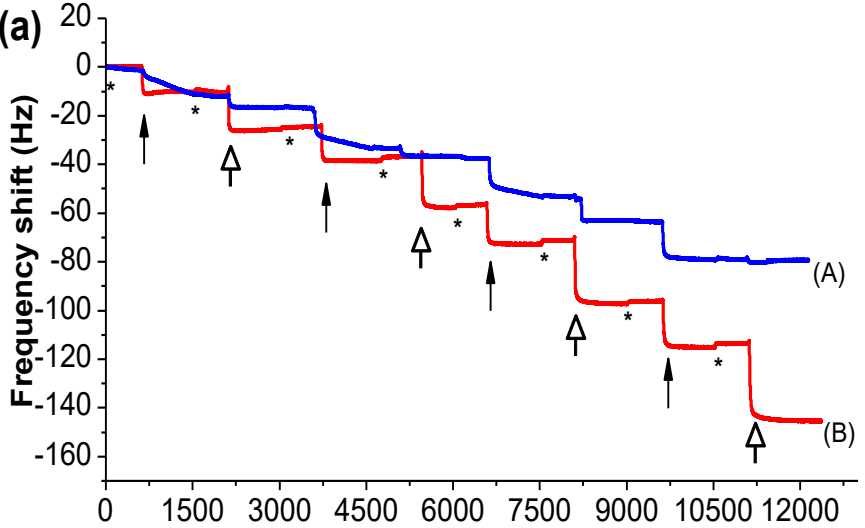

Time (s) (b)

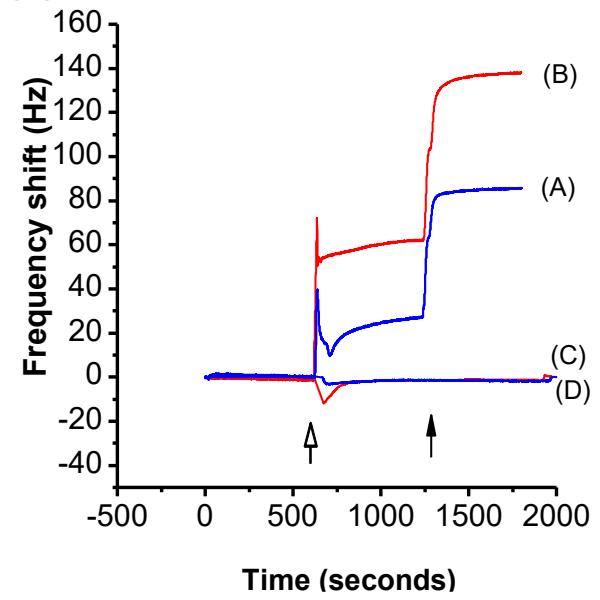

Fig. 2: (a) Monitoring of the LbL build-up of (A) PEI-ene(PVS-ene/PAH-ene) ${ }_{4}$ and (B) PEI-ene(PSS-ene/PAH-ene) ${ }_{4}$ films by insitu QCM experiments (*: rinsing step with acidic solution $(\mathrm{pH} 3)$; filled arrow: polyanion-ene addition and unfilled arrow: polycation-ene addition). (b) QCM measurements performed on gold sensors coated with: (A) uncrosslinked PEI-ene(PVSene/PAH-ene $)_{4}$; (B) uncrosslinked PEI-ene(PSS-ene/PAH-ene) $)_{4}$; (C) crosslinked PEI-ene(PVS-ene/PAH-ene) $)_{4}$ films using PEGdi-SH as a dithiol crosslinker; (D) crosslinked PEI-ene(PSS-ene/PAH-ene) $)_{4}$ films using the same crosslinker. The sample was initially placed in contact with acidic medium ( $\mathrm{pH} 3)$; after an equilibrium time (10 min.), the acidic solution was replaced by a basic solution ( $\mathrm{pH} 14$, unfilled arrow), then the sample was rinsed with acidic medium (filled arrow). 
As for conventional polyelectrolyte multilayer assemblies, the growth mechanism of PEI-ene(PVS-ene/PAH-ene)n and PEI-ene (PVS-ene/PAH-ene) ${ }_{n}$ films should result from the overcompensation of the surface charge by the adsorbed polymer layer.[14] Therefore, environmental changes such as $\mathrm{pH}$ and/or ionic strength may affect the stability of the multilayer assembly, the ultimate stage being the disassembly of the films.[48] By increasing the $\mathrm{pH}$ over the typical $\mathrm{pK}_{\mathrm{a}}$ values of amines $(\mathrm{pH}>11)$, the PAH-ene underwent deprotonation preventing the formation of electrostatic interactions with PSS-ene or PVS-ene. A spontaneous disassembly of the PAH-ene-containing multilayer films was thus expected under basic conditions. To address this crucial issue, a covalent crosslinking between the film-forming layers and between the multilayer films and the substrate was targeted through a photochemical process. The latter involved a thiol-ene radical addition between a dithiol crosslinker and $\mathrm{C}=\mathrm{C}$ double bonds on allyl-functionalized substrate and PE-enes. In a previous study, we have clearly evidenced an enhanced stability of PEM films after crosslinking, and most importantly, a higher crosslinking efficiency ofamacromolecular dithiol crosslinker (PEG-diSH) than a molecular analogue (dithiothreitol, DTT). Indeed, partial PEM film removal was only observed upon DTT as a crosslinker (10\% removal of the multilayer film). [39] Consequently, in the present work, PEI-ene(PVS-ene/PAH-ene $)_{n}$ and PEI-ene (PVS-ene/PAH-ene $)_{\mathrm{n}}$ were crosslinked by using PEG-diSH. The stability of PEM films under extreme conditions of $\mathrm{pH}(\mathrm{pH}$ 14) was then investigated by QCM monitoringfor both uncrosslinked and crosslinked assemblies (Fig. 7b).

The values of the final equilibrium frequency shift as determined from Fig. 7a after the LbL build-up were used as references to evaluate the multilayer stability versus $\mathrm{pH}$. From Fig. $7 \mathbf{b}$ (A and B), changing from an acidic to a basic medium led to a fast and steep variation of the equilibrium frequency shift of about $+85 \pm 3 \mathrm{~Hz}$ and $+137 \pm 5 \mathrm{~Hz}$ for the uncrosslinked PEI-ene(PVS-ene/PAH-ene $)_{4}$ and uncrosslinked PEI-ene(PSS-ene/PAH-ene) $)_{4}$, respectively. This suggested quantitative removal of the corresponding multilayer films from the substrate. In contrast, no significant frequency variation was evidenced for the photochemically crosslinked films, upon changing from acidic to basic conditions (Fig. 7b, C and D). These results confirmed unambiguously the efficiency of the photochemical crosslinking reaction and the robustness associated with the crosslinked multilayered films toward dramatic changes in $\mathrm{pH}$, thus suggesting enhanced long-term stability of the latter PEM films.

\subsection{Conclusion}

The controlled allylation of two types of polyelectrolytes, i.e. polycations (PAH and PEI) and strong polyanions (PSS and PVS), was critically described. The side-chain functionalization of each type presented its own drawback, i.e. heterogeneous reaction in the case of strong polyanions and chemical instability of partially protonated allylated polyamine-based polycations. On the one hand, the issue of heterogeneity associated with strong polyanions accounted for the lower ratio of allylation of PSS and PVS than that expected, although all precautions were taken into account. On the other hand, the gradual change in the solubility behavior (instability) of the partially protonated allylated polyamines was a result of both hydrogen bonding (physical interactions) and hydroamination reactions (chemical crosslinking), as thoroughly investigated by different experimental tests. The hydroamination could be considered as the main reason inducing a permanent insolubility of the partially protonated allylated polyamines, due to its ability to form covalent bonds. This conclusion was further confirmed by liquid ${ }^{1} \mathrm{H}$ RMN that evidenced a gradual decrease in the intensity of allylic protons in a model reaction between two small molecules ( $n$-butylamine and allyl bromide). Since both undesirable crosslinking reactions (hydrogen bonding and hydroamination) could take place only when partially protonated allylated polyamines were involved, the simplest solution was to store the resulting allylated polyamines under their fully protonated forms (PAH-ene and PEI-ene) before further use.

After partial allylation, all resulting PE-ene products still maintained their electrolytic properties with suitable zeta-potential values allowing for successful construction of two series of PEM films, namely PEI-ene(PSS-ene/PAHene $)_{\mathrm{n}}$ and PEI-ene(PVS-ene/PAH-ene) $)_{\mathrm{n}}$, as monitored by QCM measurements. Although PEI, PAH, PSS, and their derivatives have been widely investigated as building blocks for multilayer coating built-up, we herein presented one of the first implementation of PVS for such a purpose.

The allylic side groups on PEs-ene and on allylated substrate were used to enhance the stability of the resulting PEM films under extreme $\mathrm{pH}$ values. Such a stability was established via thiol-ene photo-crosslinking between different $\mathrm{C}=\mathrm{C}$ double bonds on $\mathrm{PE}$ layers and especially those on the allylated substrate in the presence of a water-soluble macromolecular dithiol crosslinker. The stability of the resulting crosslinked PEM films (both PEI-ene(PVS-ene/PAHene $)_{4}$ and PEI-ene(PSS-ene/PAH-ene $)_{4}$ ) under strongly alkaline conditions ( $\mathrm{pH} 14$ ) was also validated by QCM measurements. 
The presence of PVS in the newly crosslinked PEI-ene(PVS-ene/PAH-ene) $)_{n}$ films reported in this study would possibly open a new avenue for applying our covalent crosslinking approach to biomedical applications. Indeed, PVS has been recently reported to present diverse biological functions very similar to those of heparin, and it has thus enabled to stimulate cell proliferation.

\section{Acknowledgements}

Financial support from CNRS and UPEC is gratefully acknowledged. The authors also thank Mrs. S. Hamadi for her kind technical assistance.

\section{Bibliography}

1. S. Llamas, E. Guzmán, F. Ortega, N. Baghdadli, C. Cazeneuve, R. G. Rubio, G. S. Luengo. Adv. Colloid Interface Sci. 222, 461-87 (2015).

2. O. Akhlaghi, Y. Z. Menceloglu, O. Akbulut. Scientific Reports. 7, 41743 (2017).

3. H. J. Kong, S. G. Bike, V. C. Li. Cement \& Concrete Res. 36, 851-7. (2006).

4. D. J. Miller, D. R. Dreyer, C. W. Bielawski, D. R. Paul, B. D. Freeman. Angew.

5. N. Shahkaramipour, T. Tran, S. Ramanan, H. Lin. Membranes. 7, 13 (2017).

6. S Mulyati, R Takagi, A Fujii, Y Ohmukai, T Maruyama, H. Matsuyama. J. Membr. Sci.. 417-418, 137-43, (2012).

7. S. L. Patwekar, A. P. Potulwar, S. R. Pedewad, M. S. Gaikwad, S. A. Khan, A. B. Suryawanshi. Int J Pharm Pharm Res Human. 5, 97-109 (2016).

8. M. Siyawamwaya, Y. E. Choonara, D. Bijukumar, P. Kumar, L. C. Du Toit, V. Pillay. Int. J. Polym. Mater. Polym. Biomater. 64, 955-68 (2015).

9. M. Zhao, L. Li, C. Zhou, F. Heyroth, B. Fuhrmann, K. Maeder, T. Groth. Biomacromolecules. 15, 4272-80 (2014).

10. H. J. Kwon. Adv. Mater. Sci. Eng. 2014, 7 (2014).

11. H. J. Kwon, K. Yasuda, J. P. Gong, Y. Ohmiya. Macromol. Res. 22, 227-35 (2014).

12. I. M. El-Sherbiny, M. H. Yacoub. Glob Cardiol Sci Pract. 2013, 38 (2013).

13. W. Lee, J. H. Seo, H. Y. Woo. Polymer. 54, 5104-21 (2013).

14. G. Decher. Science. 277, 1232-7 (1997).

15. H. Rammal, C. Harmouch, C. Maerten, C. Gaucher, F. Boulmedais, P. Schaaf, J. C. Voegel, D. Laurent-Maquin, P. Menu, H. Kerdjoudj. J. Biomed. Mater. Res. Part A. 105, 292-300 (2017).

16. C. Vigier-Carriere, T. Garnier, D. Wagner, P. Lavalle, M. Rabineau, J. Hemmerle, B. Senger, P. Schaaf, F. Boulmedais, L. Jierry. Angew. Chem. Int. Ed. 54, 10198-201 (2015).

17. $\quad$ Z. B. Liu, L.; Zhao, G.; Liu, Y. . Beilstein J. Nanotechnol. 7, 1028-32 (2016).

18. J. Borges, J. F. Mano. Chem. Rev. 114, 8883-942 (2014).

19. S. T. Dubas, J. B. Schlenoff. Macromolecules. 34, 3736-40 (2001).

20. G. Rydzek, P. Schaaf, J.-C. Voegel, L. Jierry, F. Boulmedais. Soft Matter. 8, 9738-55 (2012).

21. Y. Tian, Q. He, C. Tao, J. Li. Langmuir. 22, 360-2 (2006).

22. Y. Hu, Y. Ding, D. Ding, M. Sun, L. Zhang, X. Jiang, C. Yang. Biomacromolecules. 8, 1069-76 (2007).

23. L. Richert, F. Boulmedais, P. Lavalle, J. Mutterer, E. Ferreux, G. Decher, P. Schaaf, J.-C. Voegel, C. Picart. Biomacromolecules. 5, 284-94 (2004).

24. C. Picart, R. Elkaim, L. Richert, F. Audoin, Y. Arntz, M. Da Silva Cardoso, P. Schaaf, J. C. Voegel, B. Frisch. Adv. Funct. Mater. 15, 83-94 (2005).

25. G. V. Martins, E. G. Merino, J. F. Mano, N. M. Alves. Macromol. Biosci. 10, 1444-55 (2010).

26. J. J. Harris, P. M. DeRose, M. L. Bruening. J. Am. Chem. Soc. 121, 1978-9 (1999).

27. L. A. Connal, C. R. Kinnane, A. N. Zelikin, F. Caruso. Chem. Mater. 21, 576-8 (2009).

28. V. Calcagno, R. Vecchione, A. Sagliano, A. Carella, D. Guarnieri, V. Belli, L. Raiola, A. Roviello, P. A. Netti. Colloids Surf. B Biointerfaces. 142, 281-9 (2016).

29. G. K. Such, J. F. Quinn, A. Quinn, E. Tjipto, F. Caruso. J. Am. Chem. Soc. 128, 9318-9 (2006).

30. J. Niu, F. Shi, Z. Liu, Z. Wang, X. Zhang. Langmuir. 23, 6377-84 (2007).

31. N. Rokhati, T. Istirokhatun, A. M. Samsudin. Int. J. renew. Energy Developpement 5, 6 (2016).

32. S. Dawlee, A. Sugandhi, B. Balakrishnan, D. Labarre, A. Jayakrishnan. Biomacromolecules. 6, 2040-8 (2005).

33. Q. An, K. Nie, Y. Zhang, Y. Wang, Y. Hu, V. Dutschk, X. Luan. Soft Matter. 11, 6859-65 (2015).

34. Y. Song, K. P. Meyers, J. Gerringer, R. K. Ramakrishnan, M. Humood, S. Qin, A. A. Polycarpou, S. Nazarenko, J. C. Grunlan. Macromol. Rapid Commun. 38, 1700064 (2017).

35.

J. Davila, A. Chassepot, J. Longo, F. Boulmedais, A. Reisch, B. Frisch, F. Meyer, J.-C. Voegel, P. J. Mésini, B. Senger, M.-H. Metz-Boutigue, J. Hemmerlé, P. Lavalle, P. Schaaf, L. Jierry. J. Am. Chem. Soc. 134, 83-6 (2012).

N. Madaan, N. Romriell, J. Tuscano, H. Schlaad, M. R. Linford. J. Colloid Interface Sci. 459, 199-205 (2015).

A. Saydjari, J. P. Long, W. J. Dressick, B. S. Simpkins. Chem. Phys. Lett. 608, 328-33 (2014).

A. Reisch, J.-C. Voegel, E. Gonthier, G. Decher, B. Senger, P. Schaaf, P. J. Mésini. Langmuir. 25, 3610-7 (2009).

39. T.-T.-T. Nguyen, S. Belbekhouche, P. Dubot, B. Carbonnier, D. Grande. Journal of Materials Chemistry A. 5, $24472-$ $83(2017)$.

40. H. Jin, J. Nam, J. Park, S. Jung, K. Im, J. Hur, J.-J. Park, J.-M. Kim, S. Kim. Chem. Commun. 47, 1758-60 (2011).

41. J. de Grooth, B. Haakmeester, C. Wever, J. Potreck, W. M. de Vos, K. Nijmeijer. Journal of Membrane Science. 489, 153-9 (2015).

42. M. E. Tagliazucchi, E. J. Calvo. Journal of Electroanalytical Chemistry. 599, 249-59 (2007).

43. F. Ji, Y. Zhang, Y. Geng, Y. Zong, L. Wang. Chemical Research in Chinese Universities. 32, 493-8 (2016).

44. T. H. Nguyen, S. J. Paluck, A. J. McGahran, H. D. Maynard. Biomacromolecules. 16, 2684-92 (2015).

45. P. Esmaeilzadeh, A. Köwitsch, F. Heyroth, G. Schmidt, S. Fischer, K. Richter, T. Groth. Carbohydrate polymers. 157, 1205-14 (2017).

46. K. Yoshida, T. Ono, Y. Kashiwagi, S. Takahashi, K. Sato, J.-i. Anzai. Polymers. 7, 1269 (2015).

47. E. Pensa, E. Cortés, G. Corthey, P. Carro, C. Vericat, M. H. Fonticelli, G. Benítez, A. A. Rubert, R. C. Salvarezza. Acc. Chem. Res. 45, 1183-92 (2012). 
48. S. S. Shiratori, M. F. Rubner. Macromolecules. 33, 4213-9 (2000). 